\title{
Achievable performance of multivariable systems with unstable zeros and poles
}

\author{
K. Havre* and S. Skogestad ${ }^{\dagger}$ \\ Chemical Engineering, Norwegian University of Science and Technology, \\ N-7491 Trondheim, Norway. \\ Submitted to International Journal of Control \\ Revised March 2001
}

\begin{abstract}
This paper examines the fundamental limitations imposed by unstable (Right Half Plane; RHP) zeros and poles in multivariable feedback systems. We generalize previously known controllerindependent lower bounds on the $\mathcal{H}_{\infty}$-norm of closed-loop transfer functions $W X V,\|W X V(s)\|_{\infty}$, where $X$ is input or output sensitivity or complementary sensitivity, to include multivariable unstable and non-minimum phase weights $W$ and $V$. The bounds are tight for cases with only one RHP-zero or pole. For plants with RHP-zeros we obtain bounds on the output performance for reference tracking and disturbance rejection. For plants with RHP-poles we obtain new bounds on the input performance. This quanitifies the minimum input usage needed to stabilize an unstable plant in the presence of disturbances or noise. For a one degree-of-freedom controller the combined effect of RHP-zeros and poles further deteriorate the output performance, whereas there is no such additional penalty with a two degrees-of-freedom controller where also the disturbance and/or reference signal is used by the controller.
\end{abstract}

Keywords: Linear system theory; Input-output controllability; Unstable systems; Right half plane zeros and poles; Stabilization; Fundamental limitations; pole vectors; zero vectors.

\section{Introduction}

It is well known that the presence of unstable RHP zeros and poles pose fundamental limitations on the achievable control performance. This was quantified for SISO systems by Bode (1945) more than 50 years ago, and most control engineers have an intuitive feeling of the limitations for scalar systems. Rosenbrock $(1966 ; 1970)$ was one of the first to point out that multivariable RHP-zeros pose similar limitations.

The main results in this paper are to provide explicit lower bounds on the $\mathcal{H}_{\infty}$-norm of closedloop transfer functions. Of course, it is relatively straightforward to compute the minimum value of the $\mathcal{H}_{\infty}$-norm for a given case by obtaining the optimal controller using standard software, e.g. in Matlab. A direct computation of the minimum value of the $\mathcal{H}_{\infty}$-norm is also possible, e.g. using the

*Present address: ABB Corporate Research, P.O.Box 90, N-1375 Billingstad, Norway. Fax: (+47) 66 84 35 41, E-mail: Kjetil.Havre@no.abb.com

†Phone: (+47) 7359 4154, Fax: (+47) 7359 4080, E-mail: skoge@ chembio.ntnu.no. 
Hankel-norm as explained in (Francis, 1987). Therefore, we want to stress that our objective is to derive explicit (analytical) bounds that yield direct insight into the fundamental limitations imposed by RHP-poles and zeros.

The basis of our results is the important work by Zames (1981), who made use of the interpolation constraint $y_{z}^{H} S(z)=y_{z}^{H}$ for a RHP-zero $z$ and the maximum modulus theorem to derive bounds on the $\mathcal{H}_{\infty}$-norm of the sensitivity function $S$ for plants with one RHP-zero. Doyle, Francis and Tannenbaum (1992) generalized this to provide bounds on both $S$ (sensitivity) and $T$ (complementary sensitivity) for plants with both RHP-zeros and RHP-poles in the SISO case, and Skogestad and Postlethwaite (1996) and Havre and Skogestad (1998) generalized this further to the MiMO case.

In this paper we extend these results by deriving lower bounds on the $\mathcal{H}_{\infty}$-norm of other important closed-loop transfer functions. To do this, we generalize the bounds on $\|W X V(s)\|_{\infty}$, where $X$ is $S$ or $T$, to include multivariable unstable and non-minimum phase weights $W$ and $V$. One important application of these bounds, is that we can quantify the minimum input usage needed for any controller to stabilize an unstable plant in the presence of "worst case" disturbance, measurement noise and reference changes.

As a motivating example, consider a single-input single-output unstable plant with a RHP-pole $p$. We want to obtain a lower bound on the $\mathcal{H}_{\infty}$-norm of the closed-loop transfer function $K_{2} S$ from measurement noise $n$ to plant input $u$. Here $K_{2}$ denotes the feedbak part of the controller. We first rewrite $K_{2} S=G^{-1} T$, which is on the form $W X V$ with $W=G^{-1}, X=T$ and $V=I$. To achieve internal stability we must satisfy the interpolation constraint $T(p)=1$, and by applying the maximum modulus principle we obtain the bound (see Theorem 3 for details)

$$
\left\|K_{2} S(s)\right\|_{\infty}=\left\|G^{-1} T(s)\right\|_{\infty} \geq\left|G_{s}^{-1}(p)\right|
$$

where $G_{s}$ is the "stable" version of $G$ (with its RHP-poles mirrored into the LHP). As an example, consider the plant $G(s)=\frac{1}{s-10}$, which has an unstable pole $p=10$. We obtain $G_{s}(s)=\frac{1}{s+10}$. For any linear feedback controller $K$, we find that the lower bound

$$
\left\|K_{2} S(s)\right\|_{\infty} \geq\left|G_{s}^{-1}(p)\right|=2 p=20
$$

must be satisfied. Thus, if we require that the plant inputs are bounded with $\|u\|_{\infty} \leq 1$, then we cannot allow the magnitude of measurement noise to exceed $\|n\|_{\infty}=1 / 20=0.05$.

An additional important contribution of this paper is to prove that the lower bounds are tight provided that plant has in many one RHP-zero and any number of RHP-poles, or one RHP-pole and any number of RHP-zeros. To prove this we derive analytical expressions for controllers that achieve the lower bound.

The presentation in this paper in brief in places, and for complete details and further examples the reader is referred to Chapter 5 in the thesis of Havre (1998).

\section{Elements from linear system theory}

\subsection{Zeros and zero directions.}

Zeros of a system arise when competing effects, internal to the system, are such that the output is zero even when the inputs and the states are not identically zero. Here we apply the following definition of zeros (MacFarlane and Karcanias, 1976).

DEFINITION 1 (ZEROS). $z_{i} \in \mathbb{C}$ is a zero of $G(s)$ if the rank of $G\left(z_{i}\right)$ is less than the normal rank of $G(s)$. 
The normal rank of $G(s)$ is defined as the rank of $G(s)$ at all $s$ except a finite number of singularities (which are the zeros). We use the following definition of the associated zero directions:

Definition 2 (ZERo DiReCTIONS). If $G(s)$ has a zero for $s=z \in \mathbb{C}$ then there exist non-zero vectors, denoted the input zero direction $u_{z} \in \mathbb{C}^{m}$ and the output zero direction $y_{z} \in \mathbb{C}^{l}$, such that $u_{z}^{H} u_{z}=1, y_{z}^{H} y_{z}=1$ and

$$
G(z) u_{z}=0 \cdot y_{z}
$$

For a system $G(s)$ with state-space realization $\left[\begin{array}{l|l}A & B \\ \hline C & D\end{array}\right]$, the zeros $z$ of the system, the input zero directions $u_{z}$ and the state input zero vectors $\boldsymbol{x}_{z i} \in \mathbb{C}^{n}$ ( $n$ is the number of states) can all be computed from the generalized eigenvalue problem

$$
\left[\begin{array}{cc}
A-s I & B \\
C & D
\end{array}\right]\left[\begin{array}{l}
\boldsymbol{x}_{z i} \\
u_{z}
\end{array}\right]=\left[\begin{array}{l}
0 \\
0
\end{array}\right]
$$

Similarly one can compute the zeros $z$ and the output zero directions $y_{z}$ from $G^{T}$.

\subsection{Poles and pole directions.}

Bode (1945) states that the poles are the singular points at which the transfer function fails to be analytic. In this work we replace "fails to be analytic" with "is infinite", which certainly implies that the transfer function is not analytic. When we evaluate the transfer function $G(s)$ at $s=p, G(p)$ is infinite in some directions at the input and the output. This is the basis for the following definition of input and output pole directions.

Definition 3 (Pole Directions). If $s=p \in \mathbb{C}$ is a distinct pole of $G(s)$ then there exist an input direction $u_{p} \in \mathbb{C}^{m}$ and an output direction $y_{p} \in \mathbb{C}^{l}$, such that $\left\|u_{p}\right\|_{2}=1$, $\left\|y_{p}\right\|_{2}=1$, and

$$
G(p) u_{p}=\infty \cdot y_{p}
$$

For a system $G(s)$ with minimal state-space realization $\left[\begin{array}{l|l}A & B \\ \hline C & D\end{array}\right]$ the pole directions $u_{p}$ and $y_{p}$ for a distinct pole $p$ can be computed from

$$
u_{p}=B^{H} x_{p i} /\left\|B^{H} x_{p i}\right\|_{2} ; \quad y_{p}=C x_{p o} /\left\|C x_{p o}\right\|_{2}
$$

where $x_{p i} \in \mathbb{C}^{n}$ and $x_{p o} \in \mathbb{C}^{n}$ are the eigenvectors corresponding to the two eigenvalue problems

$$
x_{p i}^{H} A=p x_{p i}^{H} ; \quad A x_{p o}=p x_{p o}
$$

For the sake of simplicity we only consider distinct poles in this paper. For computation and definition of pole directions in the case when the pole $p$ is repeated we refer to Havre (1998, Chapter 2).

\subsection{All-pass factorizations of RHP zeros and poles}

A transfer function matrix $B(s)$ is all-pass if $B^{T}(-s) B(s)=I$, which implies that all singular values of $B(j \omega)$ are equal to one.

\footnotetext{
${ }^{1}$ Strictly speaking, the transfer function $G(s)$ can not be evaluated at $s=p$, since $G(s)$ is not analytic at $s=p$.
} 
A rational transfer function matrix $M(s)$ with RHP-poles $p_{i} \in \mathbb{C}_{+}$, can be factorized either at the input (subscript $i$ ) or at the output (subscript $o$ ) as follows ${ }^{2}$

$$
M(s)=M_{s i} \mathcal{B}_{p i}^{-1}(M) ; \quad M(s)=\mathcal{B}_{p o}^{-1}(M) M_{s o}(s)
$$

$M_{s i}, M_{s o}-$ Stable (subscript $s$ ) versions of $M$ with the RHP-poles mirrored across the imaginary axis.

$\mathcal{B}_{p i}(M), \mathcal{B}_{p o}(M)$ - Stable all-pass rational transfer function matrices containing the RHP-poles (subscript $p$ ) of $M$ as RHP-zeros.

The all-pass filters are

$$
\begin{array}{ll}
\mathcal{B}_{p i}(M(s))=\prod_{i=1}^{N_{p}}\left(I-\frac{2 \operatorname{Re}\left(p_{i}\right)}{s+\bar{p}_{i}} \hat{u}_{p_{i}} \hat{u}_{p_{i}}^{H}\right) ; & \mathcal{B}_{p i}^{-1}(M(s))=\prod_{i=N_{p}}^{1}\left(I+\frac{2 \operatorname{Re}\left(p_{i}\right)}{s-p_{i}} \hat{u}_{p_{i}} \hat{u}_{p_{i}}^{H}\right) \\
\mathcal{B}_{p o}(M(s))=\prod_{i=N_{p}}^{1}\left(I-\frac{2 \operatorname{Re}\left(p_{i}\right)}{s+\bar{p}_{i}} \hat{y}_{p_{i}} \hat{y}_{p_{i}}^{H}\right) ; & \mathcal{B}_{p o}^{-1}(M(s))=\prod_{i=1}^{N_{p}}\left(I+\frac{2 \operatorname{Re}\left(p_{i}\right)}{s-p_{i}} \hat{y}_{p_{i}} \hat{y}_{p_{i}}^{H}\right)
\end{array}
$$

$\mathcal{B}_{\text {po }}(M)$ is obtained by factorizing at the output one RHP-pole at a time, starting with

$$
M=\mathcal{B}_{p_{1} o}^{-1}(M) M_{p_{1} o}
$$

where

$$
\mathcal{B}_{p_{1} o}^{-1}(M(s))=I+\frac{2 \operatorname{Re}\left(p_{1}\right)}{s-p_{1}} \hat{y}_{p_{1}} \hat{y}_{p_{1}}^{H}
$$

and $\hat{y}_{p_{1}}=y_{p_{1}}$ is the output pole direction of $M$ for $p_{1}$. This procedure may be continued to factor out $p_{2}$ from $M_{p_{1} o}$ where $\hat{y}_{p_{2}}$ is the output pole direction of $M_{p_{1} o}$ (which need not coincide with $y_{p_{2}}$, the pole direction ${ }^{3}$ of $M$ ) and so on. A similar procedure may be used to factorize the poles at the input of $M$. Note that the sequence get reversed in the input factorization compared to the output factorization.

In a similar sequential manner, the RHP-zeros can be factorized either at the input or at the output of $M$

$$
M(s)=M_{m i} \mathcal{B}_{z i}(M(s)) ; \quad M(s)=\mathcal{B}_{z o}(M) M_{m o}(s)
$$

$M_{m i}, M_{m o}-$ Minimum phase (subscript $m$ ) versions of $M$ with the RHP-zeros mirrored across the imaginary axis.

$\mathcal{B}_{z i}(M), \mathcal{B}_{z o}(M)$ - Stable all-pass rational transfer function matrices containing the RHP-zeros (subscript $z$ ) of $M$.

We get

$$
\begin{array}{ll}
\mathcal{B}_{z i}(M(s))=\prod_{j=N_{z}}^{1}\left(I-\frac{2 \operatorname{Re}\left(z_{j}\right)}{s+\bar{z}_{j}} \hat{u}_{z_{j}} \hat{u}_{z_{j}}^{H}\right) ; & \mathcal{B}_{z i}^{-1}(M(s))=\prod_{j=1}^{N_{z}}\left(I+\frac{2 \operatorname{Re}\left(z_{j}\right)}{s-z_{j}} \hat{u}_{z_{j}} \hat{u}_{z_{j}}^{H}\right) \\
\mathcal{B}_{z o}(M(s))=\prod_{j=1}^{N_{z}}\left(I-\frac{2 \operatorname{Re}\left(z_{j}\right)}{s+\bar{z}_{j}} \hat{y}_{z_{j}} \hat{y}_{z_{j}}^{H}\right) ; & \mathcal{B}_{z o}^{-1}(M(s))=\prod_{j=N_{z}}^{1}\left(I+\frac{2 \operatorname{Re}\left(z_{j}\right)}{s-z_{j}} \hat{y}_{z_{j}} \hat{y}_{z_{j}}^{H}\right)
\end{array}
$$

\footnotetext{
${ }^{2}$ Note that the notation on the all-pass factorizations of RHP zeros and poles used in this paper is reversed compared to the notation used in (Green and Limebeer, 1995; Skogestad and Postlethwaite, 1996). The reason for this change of notation is to be consistent with what the literature generally defines as an all-pass filter.

${ }^{3}$ In fact: $\hat{y}_{p_{2}}=\left.\mathcal{B}_{p_{1} o}^{-H}(M)\right|_{s=p_{2}} y_{p_{2}}$. Here $\left.\mathcal{B}\right|_{s=s_{0}}$ means the rational transfer function matrix $\mathcal{B}(s)$ evaluated at the complex number $s=s_{0}$. Thus, it provides an alternative to $\mathcal{B}\left(s_{0}\right)$, and it will mainly be used to avoid double parenthesis.
} 
Alternative all-pass factorizations are in use, e.g. the inner-outer factorizations used in (Morari and Zafiriou, 1989) which are the same as (9) and (10) except for the multiplication by a constant unitary matrix. Some reasons for using the factorizations given here are:

1) The factorizations of RHP-zeros are analytic and in terms of the zeros and the zero directions, whereas the inner-outer factorizations in (Morari and Zafiriou, 1989) are given in terms of the solution to an algebraic Riccati equation.

2) To factorize RHP-poles using the inner-outer factorization one needs to assume that $G^{-1}$ exist.

\subsection{Closing the loop}

In this paper we consider the general two degrees-of-freedom control configuration Figure 1. The controller can be divided into a negative feedback part $K_{2}$ from the plant measurements $y_{m}$ and a "feed forward" part $K_{1}$ from the references $r$,

$$
u=K_{1} r-K_{2} y_{m}=K_{1} r-K_{2}(y+n)
$$

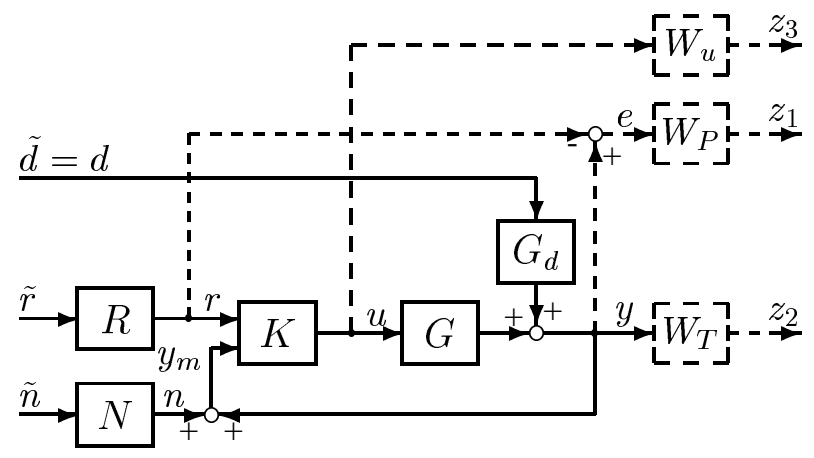

Figure 1: Two degrees-of-freedom control configuration with $K(s)=\left[K_{1}(s)-K_{2}(s)\right]$

In the figure performance weights are given in dashed boxes. The external inputs include disturbances $d$, references $r$ and measurement noise $n$ The corresponding three matrices $G_{d}, R$ and $N$ can be viewed as weights on the inputs, and the inputs $\tilde{d}, \tilde{r}$ and $\tilde{n}$ are assumed to be normalized equal to 1 in magnitude. Normally, $N$ is diagonal and $[N]_{i i}$ is the inverse of the signal to noise ratio. For most practical purposes, we can assume that $R$ and $N$ are stable. However, from a technical point of view it suffices that the unstable modes in $N$ and $R$ can be stabilized using the plant inputs $u$ and plant outputs $y$. For the disturbance plant $G_{d}$ we assume that all the unstable modes of $G_{d}$ also appear in $G$ such that they are stabilizable using the plant inputs and outputs.

The closed-loop transfer function $F$ from external inputs to external outputs,

$$
v=\left[\begin{array}{c}
\tilde{r} \\
\tilde{d} \\
\tilde{n}
\end{array}\right] \quad \text { to } \quad z=\left[\begin{array}{c}
z_{1} \\
z_{2} \\
z_{3}
\end{array}\right]^{T}=\left[\begin{array}{c}
W_{P}(y-r) \\
W_{T} y \\
W_{u} u
\end{array}\right]
$$

is

$$
F(s)=\left[\begin{array}{ccc}
W_{P}\left(S G K_{1}-I\right) R & W_{P} S G_{d} & -W_{P} T N \\
W_{T} S G K_{1} R & W_{T} S G_{d} & -W_{T} T N \\
W_{u} S_{I} K_{1} R & -W_{u} K_{2} S G_{d} & -W_{u} K_{2} S N
\end{array}\right]
$$


where the sensitivity $S$, the complementary sensitivity $T$ and the input sensitivity $S_{I}$ are defined by

$$
\begin{aligned}
S & \triangleq\left(I+G K_{2}\right)^{-1} \\
T & \triangleq I-S=G K_{2}\left(I+G K_{2}\right)^{-1} \\
S_{I} & \triangleq\left(I+K_{2} G\right)^{-1}
\end{aligned}
$$

We also define the input complementary sensitivity

$$
T_{I} \triangleq I-S_{I}=K_{2} G\left(I+K_{2} G\right)^{-1}
$$

By setting $K_{1}=K_{2}$ in the above equations, the one degree-of-freedom control configuration can be analyzed.

\section{Lower bounds on the $\mathcal{H}_{\infty}$-norm of closed-loop transfer func- tions}

In this section we derive general lower bounds on the $\mathcal{H}_{\infty}$-norm of closed-loop transfer functions when the plant $G$ has one or more RHP zeros and/or poles, by using the interpolation constraints and the maximum modulus principle. The bounds are applicable to closed-loop transfer functions on the form

$$
W(s) X(s) V(s)
$$

where we assume that $W X V$ is stable and $X$ may be $S, T, S_{I}$ or $T_{I}$. The idea is to derive lower bounds on $\|W X V(s)\|_{\infty}$ which are independent of the controller $K$. The weights $W$ and $V$ must be independent of $K$. Examples of "weights" considered in this paper are $G_{d}$ and $G^{-1}$. The weights may be unstable, provided the unstable modes can be stabilized by feedback control of the plant $G$, such that $W X V$ is stable. The results are stated in terms of four theorems.

Theorems 1 and 2 provide lower bounds on the $\mathcal{H}_{\infty}$-norm of closed-loop transfer functions on the forms $W S V$ and $W S_{I} V$ caused by one or more RHP-zeros in $G$. By maximizing over all RHP-zeros, we find the largest lower bounds on $\|W S V(s)\|_{\infty}$ and $\left\|W S_{I} V(s)\right\|_{\infty}$ which takes into account one RHP-zero and all RHP-poles in the plant.

THEOREM 1 (LOWER BOUND ON $\|W S V(s)\|_{\infty}$ ). Consider a plant G with $N_{z} \geq 1$ RHP-zeros $z_{j}$, output directions $y_{z_{j}}$ and $N_{p} \geq 0$ RHP-poles $p_{i} \in \mathbb{C}_{+}$. Let $W$ and $V$ be rational transfer function matrices, where $W$ is stable. Assume that the closed-loop transfer function WSV is (internally) stable. Then the following lower bound on $\|W S V(s)\|_{\infty}$ applies:

$$
\|W S V(s)\|_{\infty} \geq \max _{\text {RHP-zeros } z_{j} \text { in } G}\left\|W_{m o}\left(z_{j}\right) y_{z_{j}}\right\|_{2} \cdot\left\|\left.y_{z_{j}}^{H} V \mathcal{B}_{z i}^{-1}\left(\mathcal{B}_{p o}(G) V\right)\right|_{s=z_{j}}\right\|_{2}
$$

The bound is tight (equality) for the case when G has only one RHP-zero.

Proof. See Appendix.

THEOREM 2 (LOWER BOUND ON $\left\|W S_{I} V(s)\right\|_{\infty}$ ). Consider a plant $G$ with $N_{z} \geq 1$ RHP-zeros $z_{j}$, input directions $u_{z_{j}}$ and $N_{p} \geq 0$ RHP-poles $p_{i} \in \mathbb{C}_{+}$. Let $W$ and $V$ be rational transfer function matrices, where $V$ is stable. Assume that the closed-loop transfer function $W S_{I} V$ is (internally) stable. Then the following lower bound on $\left\|W S_{I} V(s)\right\|_{\infty}$ applies:

$$
\left\|W S_{I} V(s)\right\|_{\infty} \geq \max _{\text {RHP-zeros, } z_{j} \text { in } G}\left\|\left.\mathcal{B}_{z o}^{-1}\left(W \mathcal{B}_{p i}(G)\right) W\right|_{s=z_{j}} u_{z_{j}}\right\|_{2} \cdot\left\|u_{z_{j}}^{H} V_{m i}\left(z_{j}\right)\right\|_{2}
$$

The bound is tight (equality) for the case when G has only one RHP-zero. 
Theorems 3 and 4 provide lower bounds on the $\mathcal{H}_{\infty}$-norm of closed-loop transfer functions on the forms $W T V$ and $W T_{I} V$ caused by one or more RHP-poles in $G$. By maximizing over all RHP-poles, we find the largest lower bounds on $\|W T V(s)\|_{\infty}$ and $\left\|W T_{I} V(s)\right\|_{\infty}$ which takes into account one RHP-pole and all RHP-zeros in the plant.

THEOREM 3 (LOWER BOUNDS ON $\|W T V(s)\|_{\infty}$ ). Consider a plant $G$ with $N_{p} \geq 1$ RHP-poles $p_{i}$, output directions $y_{p_{i}}$ and $N_{z} \geq 0$ RHP-zeros $z_{j} \in \mathbb{C}_{+}$. Let $W$ and $V$ be rational transfer function matrices, where $V$ is stable. Assume that the closed-loop transfer function WTV is (internally) stable. Then the following lower bound on $\|W T V(s)\|_{\infty}$ applies:

$$
\|W T V(s)\|_{\infty} \geq \max _{\text {RHP-poles, } p_{i} \text { in } G}\left\|\left.\mathcal{B}_{z o}^{-1}\left(W \mathcal{B}_{z o}(G)\right) W\right|_{s=p_{i}} y_{p_{i}}\right\|_{2} \cdot\left\|y_{p_{i}}^{H} V_{m i}\left(p_{i}\right)\right\|_{2}
$$

The bound is tight (equality) for the case when G has only one RHP-pole.

THEOREM 4 (LOWER Bounds ON $\left\|W T_{I} V(s)\right\|_{\infty}$ ). Consider a plant $G$ with $N_{p} \geq 1$ RhP-poles $p_{i}$, input directions $u_{p_{i}}$ and $N_{z} \geq 0$ RHP-zeros $z_{j} \in \mathbb{C}_{+}$. Let $W$ and $V$ be rational transfer function matrices, where $W$ is stable. Assume that the closed-loop transfer function $W T_{I} V$ is (internally) stable. Then the following lower bound on $\left\|W T_{I} V(s)\right\|_{\infty}$ applies:

$$
\left\|W T_{I} V(s)\right\|_{\infty} \geq \max _{\text {RHP-poles, } p_{i} \text { in } G}\left\|W_{m o}\left(p_{i}\right) u_{p_{i}}\right\|_{2} \cdot\left\|\left.u_{p_{i}}^{H} V \mathcal{B}_{z i}^{-1}\left(\mathcal{B}_{z i}(G) V\right)\right|_{s=p_{i}}\right\|_{2}
$$

The bound is tight (equality) for the case when G has only one RHP-pole.

Remarks on Theorems 1-4:

1) The somewhat messy notation can easily be interpreted. As an example take the last factor of (18): It says that we should factorize the RHP-poles at the output of $G$ into an all-pass filter $\mathcal{B}_{p o}(G)$ (yields RHP-zeros), multiply on the right by $V$ (this may add RHP-zeros if $V$ is non-minimum phase), then factorize at the input the RHP-zeros of the product into an all-pass transfer function, take its inverse, multiply on the left by $y_{z_{j}}^{H} V$ and finally evaluate the result for $s=z_{j}$.

2) The lower bounds (18)-(21) are independent of the feedback controller $K_{2}$ if the weights $W$ and $V$ are independent of $K_{2}$.

3) The internal stability assumption on the closed-loop transfer function $W X V$, where $X \in$ $\left\{S, S_{I}, T, T_{I}\right\}$, implies that $W X V$ is stable and that we have no RHP pole/zero cancelations between the plant $G$ and the feedback controller $K_{2}$. Note that RHP pole/zero cancelations between the weights ( $W$ or $V$ ) and $X$ may be allowed.

4) The assumption on stability of $W$ and $V$ in Theorems 1-4 is in practice not restrictive, since when the assumption is not fulfilled we can generally rewrite the transfer function and apply another theorem instead. This is illustrated in the following example.

EXAMPLE 1. We want to derive a lower bound on the $\mathcal{H}_{\infty}$-norm of the closed-loop transfer function $K_{2} S G_{d}$ (input usage due to disturbances). We can use the relation $K_{2} S G_{d}=G^{-1} T G_{d}$ and apply Theorem 3 with $W=G^{-1}$ and $V=G_{d}$, but we must assume that $G_{d}$ is stable. However, we can use the relation $K_{2} S G_{d}=T_{I} G^{-1} G_{d}$ and apply Theorem 4 with $W=I$ and $V=G^{-1} G_{d}$, and in this case we can also allow $G_{d}$ to be unstable.

\section{Applications of lower bounds}

The lower bounds on $\|W X V(s)\|_{\infty}$ in Theorems 1 to 4 can be used to derive a large number of interesting and useful bounds. 


\subsection{Output performance}

The previously derived bounds in terms of the $\mathcal{H}_{\infty}$-norms of $S$ and $T$ given in (Zames, 1981; Skogestad and Postlethwaite, 1996) and in Havre and Skogestad (1998) follow easily, and further generalizations involving output performance can be derived. Here we assume that the performance weights $W_{P}$ and $W_{T}$ are stable and minimum phase.

Weighted sensitivity, $W_{P} S$. Select $W=W_{P}, V=I$, and apply the bound (18) on $W S V$ to obtain

$$
\left\|W_{P} S(s)\right\|_{\infty} \geq \max _{\text {RHP-zeros, } z_{j}}\left\|W_{P}\left(z_{j}\right) y_{z_{j}}\right\|_{2} \cdot\left\|\left.y_{z_{j}}^{H} \mathcal{B}_{p o}^{-1}(G)\right|_{s=z_{j}}\right\|_{2}
$$

Disturbance rejection. Select $W=W_{P}, V=G_{d}$, and apply the bound (18) on $W S V$ to obtain

$$
\left\|W_{P} S G_{d}(s)\right\|_{\infty} \geq \max _{\text {RHP-zeros, } z_{j}}\left\|W_{P}\left(z_{j}\right) y_{z_{j}}\right\|_{2} \cdot\left\|\left.y_{z_{j}}^{H} G_{d} \mathcal{B}_{z i}^{-1}\left(\mathcal{B}_{p o}(G) G_{d}\right)\right|_{s=z_{j}}\right\|_{2}
$$

Reference tracking. Select $W=W_{P}, V=R$, and apply the bound (18) on $W S V$ to obtain

$$
\left\|W_{P} S R(s)\right\|_{\infty} \geq \max _{\text {RHP-zeros, } z_{j}}\left\|W_{P}\left(z_{j}\right) y_{z_{j}}\right\|_{2} \cdot\left\|\left.y_{z_{j}}^{H} R \mathcal{B}_{z i}^{-1}\left(\mathcal{B}_{p o}(G) R\right)\right|_{s=z_{j}}\right\|_{2}
$$

Note that we can also look at the combined effect of disturbances and references by selecting $V=$ $\left[\begin{array}{ll}G_{d} & R\end{array}\right]$.

Measurement noise rejection. Select $W=W_{P}, V=N$ (stable), and apply the bound (20) on $W T V$ to obtain

$$
\left\|W_{P} T N(s)\right\|_{\infty} \geq \max _{\text {RHP-poles, } p_{i}}\left\|\left.\mathcal{B}_{z o}^{-1}\left(W_{P} \mathcal{B}_{z o}(G)\right) W_{P}\right|_{s=p_{i}} y_{p_{i}}\right\|_{2} \cdot\left\|y_{p_{i}}^{H} N_{m i}\left(p_{i}\right)\right\|_{2}
$$

\subsection{Input usage}

We here derive from Theorems 3 and 4 some bounds in terms of input usage. These new bounds provide very interesting insights, for example, into the possibility of stabilizing an unstable plant with inputs of bounded magnitude.

The basis for deriving these bounds is to note that the transfer function from the outputs to the inputs, $K_{2} S$, can be rewritten as $K_{2} S=T_{I} G^{-1}$ or $K_{2} S=G^{-1} T$. When $G$ is unstable, $G^{-1}$ has one or more RHP-zeros, so it is important that the bounds in Theorem 4 can handle the case when $V=G^{-1}$ has RHP-zeros. We assume that the weight $W_{u}$ on the input $u$ is stable and minimum phase.

Outputs to inputs, $K_{2} S$. Two useful lower bounds on $\left\|K_{2} S(s)\right\|_{\infty}$ can be derived. First, apply the equality $K_{2} S=T_{I} G^{-1}$, select $W=I, V=G^{-1}$, and use the bound (21) on $W T_{I} V$ to obtain

$$
\left\|K_{2} S(s)\right\|_{\infty} \geq \max _{\text {RHP-poles, } p_{i}}\left\|\left.u_{p_{i}}^{H} G^{-1} \mathcal{B}_{z i}^{-1}\left(G_{m i}^{-1}\right)\right|_{s=p_{i}}\right\|_{2}=\left\|\left.u_{p_{i}}^{H} G_{s o}^{-1}\right|_{s=p_{i}}\right\|_{2}
$$

where the last identity follows from $\mathcal{B}_{z i}\left(G_{m i}^{-1}\right)=\mathcal{B}_{z i}\left(G^{-1}\right)=\mathcal{B}_{p o}(G)$ and $G^{-1} \mathcal{B}_{p o}^{-1}(G)=G_{s o}^{-1}$.

Similarly, we obtain from the bound (20) on $W T V$, with $W=G^{-1}$ and $V=I$

$$
\left\|K_{2} S(s)\right\|_{\infty} \geq \max _{\text {RHP-poles, } p_{i}}\left\|\left.\mathcal{B}_{z o}^{-1}\left(G_{m o}^{-1}\right) G^{-1}\right|_{s=p_{i}} y_{p_{i}}\right\|_{2}=\left\|\left.G_{s i}^{-1}\right|_{s=p_{i}} y_{p_{i}}\right\|_{2}
$$

where the last identity follows from $\mathcal{B}_{z o}\left(G_{\text {mo }}^{-1}\right)=\mathcal{B}_{z o}\left(G^{-1}\right)=\mathcal{B}_{p i}(G)$. 
Disturbances to weighted inputs This generalizes the bound (26). Apply the equality $K_{2} S=$ $T_{I} G^{-1}$, select $W=W_{u}, V=G^{-1} G_{d}$, and use the bound (21) on $W T_{I} V$ to obtain

$$
\begin{aligned}
& \left\|W_{u} K_{2} S G_{d}(s)\right\|_{\infty} \geq \max _{\text {RHP-poles, } p_{i}} \\
& \quad\left\|W_{u}\left(p_{i}\right) u_{p_{i}}\right\|_{2} \cdot\left\|\left.u_{p_{i}}^{H} G^{-1} G_{d} \mathcal{B}_{z i}^{-1}\left(G_{m i}^{-1} G_{d}\right)\right|_{s=p_{i}}\right\|_{2}
\end{aligned}
$$

where we have used the identity $\mathcal{B}_{z i}(G) G^{-1}=G_{m i}^{-1}$.

Noise and references to weighted inputs. To consider noise to weighted inputs replace $G_{d}$ by $N$ in (28). To consider references to weighted inputs for the case of one-degree of freedom control replace $G_{d}$ by $R$ in (28). We may look at the combined effect of reference tracking, disturbance rejection and measurement noise by using the bound (21) on $W T_{I} V$ with $W=W_{u}$ and $V=G^{-1}\left[\begin{array}{lll}G_{d} & R & N\end{array}\right]$.

\section{Two degrees-of-freedom control}

For a two degrees-of-freedom controller the closed-loop transfer function from references $\tilde{r}$ to outputs $z_{1}=W_{p}(y-r)$ becomes

$$
W_{P}\left(S G K_{1}-I\right) R
$$

We then have the following lower bound which does not follow from Theorems 1-4:

THEOREM 5. Consider a plant $G$ with $N_{z} \geq 1$ RHP-zeros $z_{j}$ and $N_{p} \geq 0$ RHP-poles $p_{i} \in \mathbb{C}_{+}$. Let the performance weight $W_{P}$ be minimum phase and let (for simplicity) $R$ be stable. Assume that the closed-loop transfer function $W_{P}\left(S G K_{1}-I\right) R$ is stable. Then the following lower bound on $\left\|W_{P}\left(S G K_{1}-I\right) R(s)\right\|_{\infty}$ applies:

$$
\left\|W_{P}\left(S G K_{1}-I\right) R(s)\right\|_{\infty} \geq \max _{\text {RHP-zeros } z_{j} \text { in } G}\left\|W_{P}\left(z_{j}\right) y_{z_{j}}\right\|_{2} \cdot\left\|y_{z_{j}}^{H} R_{m i}\left(z_{j}\right)\right\|_{2}
$$

The bound (30) is tight if the plant has only one RHP-zero.

The bound in (30) should be compared to the following bound for a one degree-of-freedom controller (which follows from Theorem 1 assuming that $W_{P}$ is minimum phase).

$$
\left\|W_{P} S R(s)\right\|_{\infty} \geq \max _{\text {RHP-zeros } z_{j} \text { in } G}\left\|W_{P}\left(z_{j}\right) y_{z_{j}}\right\|_{2} \cdot\left\|\left.y_{z_{j}}^{H} R \mathcal{B}_{z i}^{-1}\left(\mathcal{B}_{p o}(G) R\right)\right|_{s=z_{j}}\right\|_{2}
$$

We note from (30) that for the two degrees-of-freedom controller only the RHP-zeros pose limitations on output performance. Thus, unlike the bound for a one degree-of-freedom controller in (31), there is no additional penalty when we have RHP-poles.

\section{Example}

Consider the following multivariable plant $G$,

$$
G(s)=\left[\begin{array}{cc}
\frac{s-z}{s-p} & -\frac{0.1 s+1}{s-p} \\
\frac{s-z}{0.1 s+1} & 1
\end{array}\right], \quad \text { with } \quad z=2.5 \text { and } p=2
$$

The plant $G$ has one multivariable RHP-zero $z=2.5$ and one RHP-pole $p=2$. The corresponding input and output zero and pole directions are

$$
u_{z}=\left[\begin{array}{l}
1 \\
0
\end{array}\right], \quad y_{z}=\left[\begin{array}{l}
0.371 \\
0.928
\end{array}\right], \quad u_{p}=\left[\begin{array}{l}
0.385 \\
0.923
\end{array}\right], \quad y_{p}=\left[\begin{array}{l}
1 \\
0
\end{array}\right]
$$


The RHP-pole $p$ can be factorized into $G(s)=\mathcal{B}_{p o}^{-1}(G) G_{s o}(s)$ where

$$
\mathcal{B}_{p o}(G)=\left[\begin{array}{cc}
\frac{s-p}{s+p} & 0 \\
0 & 1
\end{array}\right] \text { and } G_{s o}(s)=\left[\begin{array}{cc}
\frac{s-z}{s+p} & -\frac{0.1 s+1}{s+p} \\
\frac{s-z}{0.1 s+1} & 1
\end{array}\right]
$$

1. Consider first the bound on output sensitivity $S$. From (18) with $W=I$ and $V=I$, we find

$$
\|S(s)\|_{\infty} \geq\left\|\left.y_{z}^{H} \mathcal{B}_{p o}^{-1}(G)\right|_{s=z}\right\|_{2}=\left\|\left[\begin{array}{ll}
0.371 & 0.928
\end{array}\right]\left[\begin{array}{ll}
9 & 0 \\
0 & 1
\end{array}\right]\right\|_{2}=3.4691
$$

To confirm that the bound is tight, we use Theorem 6 in Appendix (with $W=I$ and $V=I$ ) to find the feedback controller which minimizes $\|S(s)\|_{\infty}$. With $k_{0}=10^{-2}$ we get the following balanced minimal state-space realization of the feedback controller $K_{2}$

$$
K_{2}(s)=G_{s m o}^{-1} P Q^{-1}(s) \stackrel{s}{=}\left[\begin{array}{c|cc}
-10 & 188.4 & -75.49 \\
\hline 0 & 306 & -122.6 \\
203 & -6508 & 2605
\end{array}\right] \quad \text { which indeed achieves } \quad\|S(s)\|_{\infty}=3.4691
$$

2. Next, we consider input usage in terms of the transfer function from outputs to inputs, $K_{2} S$. We have two lower bounds on $\left\|K_{2} S(s)\right\|_{\infty}$, but they are identical since the bounds are tight when the plant has only one RHP-pole. From (26) we get

$$
\left\|K_{2} S(s)\right\|_{\infty} \geq\left\|\left.u_{p}^{H} G^{-1} \mathcal{B}_{z i}^{-1}\left(G_{m i}^{-1}\right)\right|_{s=p}\right\|_{2}=\left\|u_{p}^{H} G_{s o}^{-1}(p)\right\|_{2}=3.077
$$

3. Let us finally consider reference tracking in terms of the transfer function $T_{r}$ from $r$ to weighted errors, $W_{P}(y-r)$. We have $R=I$ and choose the performance weight

$$
W_{P}(s)=w_{P}(s) \cdot I, \quad \text { with } \quad w_{P}(s)=\frac{s / 2+0.5}{s}
$$

which requires integral action, a bandwidth of 0.5 [rad/s], and a sensitivity peak less than 2 at high frequencies. With a one degree-of-freedom controller (where $K_{1}=K_{2}$ ) we get from (22) that

$$
\left\|T_{r}(s)\right\|_{\infty}=\left\|W_{P} S(s)\right\|_{\infty}=\left\|W_{P}(z) y_{z}\right\|_{2} \cdot\left\|\left.y_{z}^{H} \mathcal{B}_{p o}^{-1}(G)\right|_{s=z}\right\|_{2}=0.7 \cdot 3.4691=2.4284
$$

With a two degrees-of-freedom controller (where $K_{1}$ is free to choose) the RHP-pole does not imply a fundamental limitation, and we get from (30) that

$$
\left\|T_{r}(s)\right\|_{\infty}=\left\|W_{P}\left(S G K_{1}-I\right) R(s)\right\|_{\infty}=\left\|W_{P}(z) y_{z}\right\|_{2} \cdot\left\|y_{z}^{H} R_{m i}(z)\right\|_{2}=0.7 \cdot 1=0.7
$$

In (Havre, 1998) more details are presented including state-space realizations for $K_{1}$ and $K_{2}$.

\section{Conclusion}

- We have derived lower bounds on the $\mathcal{H}_{\infty}$-norm of closed-loop transfer functions for multivariable plants. The bounds are independent of the controller and therefore reflects the input-output controllability of the plant.

- The lower bounds are tight when the plant has only one RHP-zero or pole.

- The bounds extend and generalize the results by Zames (1981), Doyle et al. (1992), Skogestad and Postlethwaite (1996) and the results given in Havre and Skogestad (1998), to also handle non-minimum phase and unstable weights. This allows us to derive new lower bounds on input usage due to disturbances, measurement noise and reference changes.

- The new lower bounds on input usage make it possible to quantify the minimum input usage for stabilization of unstable plants in the presence of disturbances, measurement noise and reference changes.

- Theorem 5 expresses the benefit of applying a two degrees-of-freedom controller compared to a one degree-of-freedom controller when the plant is unstable and has a RHP-zero. 


\section{References}

Bode, H. W. (1945). Network Analysis and Feedback Amplifier Design, D. Van Nostrand Co., New York.

Boyd, S. and Desoer, C. A. (1985). Subharmonic functions and performance bounds in linear timeinvariant feedback systems, IMA J. Math. Contr. and Info. 2: 153-170.

Chen, J. (1995). Sensitivity integral relations and design trade-offs in linear multivariable feedback systems, IEEE Transactions on Automatic Control AC-40(10): 1700-1716.

Doyle, J. C., Francis, B. and Tannenbaum, A. (1992). Feedback Control Theory, Macmillan Publishing Company.

Francis, B. A. (1987). A course in $\mathcal{H}_{\infty}$ control theory, Lecture Notes in Control and Information Sciences, Springer-Verlag, Berlin.

Green, M. and Limebeer, D. J. N. (1995). Linear Robust Control, Prentice-Hall, Englewood Cliffs.

Havre, K. (1998). Studies on Controllability Analysis and Control Structure Design, PhD thesis, Norwegian University of Science and Technology, Trondheim.

Havre, K. and Skogestad, S. (1998). Effect of RHP zeros and poles on performance in multivariable systems, Journal of Process Control 8(3): 155-164.

MacFarlane, A. G. J. and Karcanias, N. (1976). Poles and zeros of linear multivariable systems: A survey of algebraic, geometric and complex variable theory, International Journal of Control 24(1): 33-74.

Morari, M. and Zafiriou, E. (1989). Robust Process Control, Prentice-Hall, Englewood Cliffs.

Rosenbrock, H. H. (1966). On the design of linear multivariable systems, Third IFAC World Congress. Paper 1A.

Rosenbrock, H. H. (1970). State-space and Multivariable Theory, Nelson, London.

Skogestad, S. and Postlethwaite, I. (1996). Multivariable Feedback Control, Analysis and Design, John Wiley \& Sons, Chichester.

Zames, G. (1981). Feedback and optimal sensitivity: model reference transformations, multiplicative seminorms, and approximate inverses, IEEE Transactions on Automatic Control AC-26(2): 301320.

The $\mathrm{PhD}$ thesis of Havre (1998) and MATLAB-software to compute the bounds are available on the Internet: http: //www. chembio.ntnu.no/users/skoge. 


\section{A Proof of Theorem 1}

We prove (18) by applying the following six steps:

1) Factor out the RHP-zeros in $W S V$ : the RHP-poles in $G$ appears as RHP-zeros in $S$. Factor out $S=\widetilde{S} \mathcal{B}_{p o}(G)$ to obtain

$$
\begin{aligned}
W S V(s) & =\mathcal{B}_{z o}(W) W_{m o} \widetilde{S} \mathcal{B}_{p o}(G) V \\
& =\mathcal{B}_{z o}(W) \underbrace{W_{m o} \widetilde{S}\left(\mathcal{B}_{p o}(G) V\right)_{m i}}_{(W S V)_{m}} \mathcal{B}_{z i}\left(\mathcal{B}_{p o}(G) V\right)
\end{aligned}
$$

$W S V$ is stable by assumption. From the assumption on internal stability it follows that $S$ is stable (if one closed-loop transfer function is stable then internal stability implies that all the other closed-loop transfer functions are stable). Then it is only the RHP-zeros in $S$ that can cancel RHP-poles in $V$ and $W$. Thus, factorizing the zeros in $\mathbb{C}_{+}$of $W$ does not introduce instability in $(W S V)_{m}$, since none of these cancel unstable modes in $S$ or $V$. Similarly, we can factorize the zeros in $\mathbb{C}_{+}$of $V$. However, when factorizing the zeros in $S$ we must avoid factorizing the zeros which cancel poles in $\mathbb{C}_{+}$of $V$. Otherwise, $(W S V)_{m}$ becomes unstable. By factorizing only the zeros in a minimal realization of $\mathcal{B}_{p o}(G) V$ we accomplish this. Since $W$ is stable there are no cancellations against the zeros in $S$ due to poles in $G$. It then follows that $(W S V)_{m}$ is stable.

2) Introduce $f(s)=\max _{\left\|x_{1}\right\|_{2}=1,\left\|x_{2}\right\|_{2}=1} x_{1}^{H}(W S V)_{m} x_{2}$, then

$$
\|W S V(s)\|_{\infty}=\left\|(W S V(s))_{m}\right\|_{\infty} \geq\|f(s)\|_{\infty}
$$

3) Apply the maximum modulus theorem to $f(s)$ at the RHP-zeros $z_{j}$ of $G$

$$
\|f(s)\|_{\infty} \geq\left|f\left(z_{j}\right)\right|
$$

4) Resubstitute the factorization of RHP-zeros in $S$, i.e. use $\widetilde{S}=S \mathcal{B}_{p o}^{-1}(G)$

$$
\begin{aligned}
f\left(z_{j}\right) & =\left.\max _{\left\|x_{1}\right\|_{2}=1,\left\|x_{2}\right\|_{2}=1} x_{1}^{H} W_{m o} S \mathcal{B}_{p o}^{-1}(G)\left(\mathcal{B}_{p o}(G) V\right)_{m i}\right|_{s=z_{j}} x_{2} \\
& =\left.\max _{\left\|x_{1}\right\|_{2}=1,\left\|x_{2}\right\|_{2}=1} x_{1}^{H} W_{m o} S V \mathcal{B}_{z i}^{-1}\left(\mathcal{B}_{p o}(G) V\right)\right|_{s=z_{j}} x_{2}
\end{aligned}
$$

5) Use the interpolation constraint for RHP-zeros $z_{j}$ in $G$, i.e. use $y_{z_{j}}^{H} S\left(z_{j}\right)=y_{z_{j}}^{H}$

$$
\begin{aligned}
f\left(z_{j}\right) & =\left.\max _{\left\|x_{1}\right\|_{2}=1,\left\|x_{2}\right\|_{2}=1} x_{1}^{H} W_{m o} S V \mathcal{B}_{z i}^{-1}\left(\mathcal{B}_{p o}(G) V\right)\right|_{s=z_{j}} x_{2} \\
& \geq\left.\max _{\left\|x_{1}\right\|_{2}=1,\left\|x_{2}\right\|_{2}=1} x_{1}^{H} W_{m o} y_{z_{j}} y_{z_{j}}^{H} S V \mathcal{B}_{z i}^{-1}\left(\mathcal{B}_{p o}(G) V\right)\right|_{s=z_{j}} x_{2} \\
& =\left.\max _{\left\|x_{1}\right\|_{2}=1,\left\|x_{2}\right\|_{2}=1} x_{1}^{H} W_{m o} y_{z_{j}} y_{z_{j}}^{H} V \mathcal{B}_{z i}^{-1}\left(\mathcal{B}_{p o}(G) V\right)\right|_{s=z_{j}} x_{2}
\end{aligned}
$$

6) Evaluate the lower bound

$$
\|W S V(s)\|_{\infty} \geq\left|f\left(z_{j}\right)\right| \geq\left\|W_{m o}\left(z_{j}\right) y_{z_{j}}\right\|_{2} \cdot\left\|\left.y_{z_{j}}^{H} V \mathcal{B}_{z i}^{-1}\left(\mathcal{B}_{p o}(G) V\right)\right|_{s=z_{j}}\right\|_{2}
$$

Since this bound applies to all RHP-zeros $z_{j}$ in $G$, the bound (18) follows.

We prove the tightness of the lower bound for the case when $G$ has only one RHP-zero by constructing controllers that achieve the bound. This may be formulated in the following theorem. 
Theorem 6 (CONTROller Which Minimizes $\|W S V(s)\|_{\infty}$ ). Consider a plant $G$ with one RhPzero $z$, output direction $y_{z}$, and $N_{p} \geq 0$ RHP-poles $p_{i} \in \mathbb{C}_{+}$. Let $W$ and $V$ be rational transfer function matrices, where $W$ is stable. A feedback controller (possible improper) which stabilizes $W S V$, is given by

$$
K_{2}(s)=G_{s m o}^{-1}(s) P(s) Q^{-1}(s)
$$

where

$$
\begin{aligned}
Q(s) & =\left.W_{m o}^{-1}(s) W_{m o}(z) V_{0} \mathcal{B}_{p o}^{-1}(G)\right|_{s=z} M_{m i}(z) M_{m i}^{-1}(s) \\
P(s) & =\mathcal{B}_{z o}^{-1}\left(G_{s o}\right)\left(I-\mathcal{B}_{p o}(G) Q\right) \\
V_{0} & =y_{z} y_{z}^{H}+k_{0}^{2} U_{0} U_{0}^{H} \quad \text { and } \quad M_{m i}(s)=\left(\mathcal{B}_{p o}(G) V(s)\right)_{m i}
\end{aligned}
$$

where the columns of the matrix $U_{0} \in \mathbb{R}^{l \times(l-1)}$ together with $y_{z}$ forms an orthonormal basis for $\mathbb{R}^{l}$ and $k_{0}$ is any constant. $P(s)$ is stable since the RHP-zero for $s=z$ in $I-\mathcal{B}_{\text {po }}(G) Q$ cancels the RHP-pole for $s=z$ in $\mathcal{B}_{z o}^{-1}\left(G_{s o}\right)$, in a minimal realization of $P$.

With this controller we have

$$
\lim _{k_{0} \rightarrow 0}\|W S V(s)\|_{\infty}=\left\|W_{m o}(z) y_{z}\right\|_{2} \cdot\left\|\left.y_{z}^{H} V \mathcal{B}_{z i}^{-1}\left(\mathcal{B}_{p o}(G) V\right)\right|_{s=z}\right\|_{2}
$$

which completes the proof of Theorem 1. 